\section{RMD Open}

Rheumatic \&

Musculoskeletal Diseases

To cite: López-Medina C, Molto A. Comorbidity management in spondyloarthritis. RMD Open 2020;6:e001135. doi:10.1136/ rmdopen-2019-001135

Received 15 April 2020 Revised 13 August 2020 Accepted 16 August 2020
Check for updates

(c) Author(s) (or their employer(s)) 2020. Re-use permitted under CC BY-NC. No commercial re-use. See rights and permissions. Published by BMJ.

${ }^{1}$ Rheumatology Department, Hôpital Cochin - APHP Centre, Paris, France

${ }^{2}$ ECAMO Team - INSERM (U1153) Clinical Epidemiology and Biostatistics PRES Sorbonne Paris-Cité Université De Paris, Paris, France

${ }^{3}$ Maimónides Biomedical Research Institute of Córdoba (IMIBIC), Córdoba, Spain ${ }^{4}$ Rheumatology Department, Reina Sofía University Hospital, Córdoba, Spain

Correspondence to

Anna Moltó;

anna.molto@aphp.fr

\title{
Comorbidity management in spondyloarthritis
}

\section{ABSTRACT}

Comorbidities are defined as coexistent clinical disorders that appear as a consequence of persistent inflammatory activity and/or treatment. Comorbidities in spondyloarthritis $(\mathrm{SpA})$ are frequent, contributing to a poorer quality of life, higher mortality and incremented healthcare costs. Several recommendations for the screening and management of these comorbidities have been developed in recent years with the aim of improving the different outcomes in these patients. Osteoporosis is the most prevalent comorbidity in patients with SpA, mainly caused by systemic inflammation and a lack of mobility, while cardiovascular diseases explain the increased mortality in patients with SpA with regard to the general population. Data from randomised controlled trials show a low incidence of infections in both patients with and without immunosuppressive treatment, and no evidence of a high incidence of malignant diseases has been demonstrated in these patients. Finally, concomitant fibromyalgia deserves attention, since its coexistence with $\mathrm{SpA}$ leads to a poorer treatment response and more switches of anti-TNF treatments. In this review, we show an update of the most common comorbidities in patients with $\mathrm{SpA}$, and we discuss the latest evidence on the management of such comorbidities.

\section{INTRODUCTION}

Spondyloarthritis (SpA) is a heterogeneous inflammatory rheumatic disease that can involve both the axial skeleton and sacroiliac joints, but patients can also present with peripheral symptoms, including arthritis, enthesitis or dactylitis, as well as extramusculoskeletal manifestations (uveitis, psoriasis and inflammatory bowel disease (IBD)). ${ }^{1}$

SpA features usually appear as a consequence of the activation of inflammatory pathways triggered by gut dysbiosis, mechanical stress and genetic factors. However, during the clinical course of the disease, patients with SpA may also suffer from other coexistent clinical disorders that appear as a consequence of persistent inflammatory activity and/or treatment (named 'comorbidities'), ${ }^{2}$ that is, manifestations with aetiopathogenic mechanisms different from

\section{Key messages}

What is already known about this subject?

- An increased prevalence of comorbidities in Spondyloarthritis (SpA) patients than in the general population has been described, leading to a greater functional impairment, poorer quality of life, excess work disability and mortality.

What does this study add?

- In this review, we show an update of the most common comorbidities in SpA patients, and we discuss the latest evidence on the management of such comorbidities.

How might this impact on clinical practice?

- Osteoporosis is the most frequent comorbidity in SpA patients, mainly explained by disease activity.

- Screening recommendations for patients with SpA are identical to the general population, except for inflammatory bowel disease-associated colorectal cancer and skin cancer.

- Specific recommendations for vaccination for patients with rheumatic diseases should be applied.

those of rheumatic disease (figure 1). In both SpA features and comorbidities, genetics plays a key role in the development of such disorders.

An increased prevalence of comorbidities in patients with $\mathrm{SpA}$ than in the general population has been described, ${ }^{3}$ leading to a greater functional impairment, poorer quality of life, excess work disability and mortality. ${ }^{4}$ This high impact of comorbidities in SpA (and in rheumatic diseases in general) resulted in the publication of the EULAR recommendations for reporting, screening and preventing comorbidities in chronic inflammatory rheumatic diseases in clinical practice. ${ }^{6}$

In this review, we will describe these comorbidities (grouped in osteoporosis, cardiovascular diseases (CVDs), infections, malignant diseases and fibromyalgia $(\mathrm{FM}))$, their risk factors and management. 


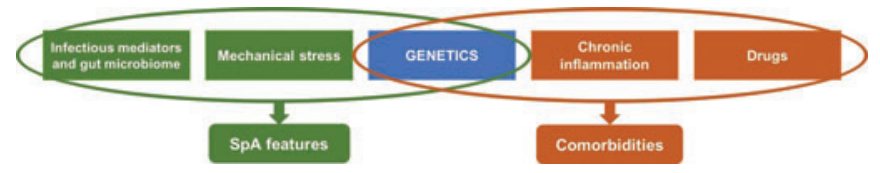

Figure 1 Spondyloarthritis features versus comorbidities.

\section{OSTEOPOROSIS}

Osteoporosis is the most frequent comorbidity among patients with SpA, with a prevalence of $13.4 \%$ (95\% CI 12.3 to 14.4$).{ }^{3}$ Causes of low bone mineral density (BMD) in patients with SpA seem to be different than in the general population, since SpA typically affects young men and glucocorticoids are rarely used (except in patients with IBD or peripheral articular involvement). ${ }^{7}$ In patients with $\mathrm{SpA}$, the main risk factors associated with low BMD are inflammation as imaged by MRI and systemic inflammation evaluated by Creactive protein (CRP) or erythrocyte sedimentation rate $^{8}$; however, baseline inflammation parameters do not determine longterm bone loss. ${ }^{910}$ The association between inflammation and low BMD in SpA was confirmed through the study of the potential positive bone effect of anti-inflammatory drugs. Briot et al demonstrated that patients receiving anti-tumour necrosis factor (anti-TNF) therapy showed a significant increase in BMD in the lumbar spine over 2 years of follow-up in comparison with patients not receiving anti-TNF therapy. ${ }^{9}$ Moreover, patients not receiving anti-TNF therapy showed a decrease in hip $\mathrm{BMD}$, in contrast with patients receiving anti-TNF therapy. The rationale of this association could be the role of TNF-alpha in bone resorption and formation, since TNFalpha enhances osteoclast activity and inhibits osteoclast apoptosis. $^{71}$ However, this beneficial effect of antiinflammatory therapy on BMD could also be explained by the amelioration of mobility-related to pain relief and increased activity. ${ }^{5}$

\section{Vertebral fractures (VFs)}

The prevalence of vertebral fractures (VFs) among the SpA population is controversial, and it has been reported to be between $6 \%$ and $20 \%$, depending on the cohort. ${ }^{12-15}$ However, this prevalence is lower in recent axial SpA cohorts, in which this prevalence is approximately $3.0 \%$ with an incidence of new VF of $1.1 \%$ over 5 years of follow-up. ${ }^{16}$ The majority of VFs in these patients are located at the level of the thoracic spine, which is also the most frequent location of vertebral deformities (due to anterior corner erosions, squaring and wedging).

Spinal fractures are a different entity and should be distinguished from VFs, since they are not related to low BMD and should not be considered a comorbidity. They can occur after a trauma in patients with an ankylosed spine, and their hallmark is an injured posterior osteoligamentous component seen in MRI or in CT. ${ }^{17}$

\section{Management of osteoporosis}

To date, there are no specific recommendations for osteoporosis management in patients with SpA. French experts in comorbidities and rheumatic diseases developed some recommendations taking into account the current state of knowledge in the field. ${ }^{18}$ These recommendations stated that a patient with a rheumatic inflammatory disease should have at least once an assessment of their bone mass densitometry. Dual-energy X-ray absorptiometry (DXA) is the most common method to evaluate BMD in the spine and hip. However, syndesmophytes may lead to an artificial increase in BMD in the lumbar spine in patients with SpA. For this reason, hip DXA is suggested as a preferred method to evaluate BMD in patients with lumbar syndesmophytes. ${ }^{19}$

In 2016, Briot et al recommended the use of available guidelines for osteoporotic patients in patients with $\mathrm{SpA}$ with severe osteoporosis and/or prevalent fractures, while standard anti-inflammatory treatment such anti-TNF (if indicated) can be used in patients without any prevalent non-traumatic fractures. ${ }^{19} \mathrm{In}$ 2009, the Canadian Dermatology-Rheumatology Comorbidity Initiative group developed practical recommendations for managing comorbidities (including osteoporosis) in patients with rheumatoid arthritis (RA), psoriatic arthritis (PsA) and cutaneous psoriasis. ${ }^{20}$ This group recommended using standardised tools/risk profiling in the assessment of osteoporosis risk in these patients. Moreover, the evaluation for fracture risk should be considered in male patients with $\mathrm{SpA}$, older than 50 years but also younger with persistent active disease, long disease duration, low BMI and ankyloses, since this is the profile of SpA patients with a high risk of fracture. ${ }^{19}$

In conclusion, in patients without any prevalent nontraumatic fracture, controlling inflammation would be the first option in the management of low BMD, while specific anti-osteoporotic drugs should be used only in patients with severe osteoporosis and/or prevalent fractures.

\section{CARDIOVASCULAR DISEASE}

The term CVD refers to ischaemic heart disease (IHD), cerebrovascular disease, peripheral vascular disease and venous thromboembolism (VTE), while major adverse cardiac events include acute myocardial infarction (MI) and cerebrovascular accident (CVA) ${ }^{21}$ Increased mortality in patients with SpA with regard to the general population has been described, which is largely explained by the higher incidence of CVD in these patients. Patients with axSpA have shown an increased risk for cardiovascular and cerebrovascular mortality in comparison with the general population. A Swedish population-based cohort study showed an increased risk of all-cause mortality in patients with ankylosing spondylitis (AS) adjusted for age and sex (HR 1.60, 95\% CI 1.44 to 1.77) in comparison with the general population, with CVD being the major 
cause of death. ${ }^{22}$ Similarly, another large populationbased study conducted in Canada showed an HR for vascular death (cardiovascular or cerebrovascular) in AS of 1.36 (95\% CI 1.13 to 1.65$)$ in comparison with controls, while this increased risk was more important in men than in women (HR 1.46 vs HR 1.24, respectively). ${ }^{23}$ Both studies found that mortality predictors were male gender, older age, lower level of education and the presence of classic CV risk factors, such as diabetes mellitus or kidney disease.

Atherosclerosis is considered one of the most important causes of increased mortality in patients with SpA, with inflammation playing a key role in the acceleration of atherogenesis. Chronic inflammatory status can contribute to the development of atheromatous plaques in all stages, from early atheroma formation to plaque instability and finally thrombus formation, with the subsequent vascular event. ${ }^{24}$ In many cases, these atheromatous plaques may be subclinical and be detected in people without clinically evident CVD. In fact, González-Juanetey and collaborators demonstrated that patients with AS without CVD showed a high prevalence of subclinical macrovascular atherosclerotic disease with an increase in carotid intima-media thickness and carotid plaques, being more severe in patients with higher disease activity and supporting the association between CVD and inflammation. ${ }^{24}$

Ischaemic heart disease (IHD) and myocardial infarction (MI) A worldwide study in patients with SpA showed an IHD global prevalence of $2.7 \%$ (95\% CI 2.2 to 3.2 ), with this prevalence differing between countries and geographic areas. ${ }^{25}$ A recent study showed a relative risk for a first acute coronary syndrome in patients with AS of $1.3(95 \%$ CI 1.0 to 1.7 ) compared with the general population, being slightly lower than that in patients with RA, who showed an HR of 1.7 (95\% CI 1.4 to 2.1$).{ }^{26}$ Patients with PsA have also shown an increased risk for MI in those using and not using disease-modifying anti-rheumatic drugs (DMARDs) (HR 1.36, 95\% CI 1.04 to 1.77 and HR $1.36,95 \%$ CI 1.01 to 1.84 , respectively). ${ }^{27}$ This increased risk of IHD and MI may be the consequence of an interplay among traditional CV risk factors, systemic inflammation and treatment characteristics, in which the use of non-steroidal anti-inflammatory drugs (NSAIDs) represents the cornerstone treatment in patients with SpA.

\section{Other cardiovascular manifestations}

Stroke or CVA show a prevalence of $1.3 \%$ (95\% CI 0.9 to 1.7 ) in the worldwide SpA population. ${ }^{3}$ Stroke incidence has been widely studied in the Swedish Population National Patient Register, showing an incidence rate of 5.4 and 5.9 stroke events per 1000 person-years at risk in patients with AS and PsA, respectively, compared with 4.7 in the general population. ${ }^{28}$ This represents an increased risk of 1.25 (95\% CI 1.06 to 1.48 ) and 1.34 (95\% CI 1.22 to 1.48) for patients with AS and PsA in comparison with the general population, although another study suggested that this higher risk in PsA is not as evident after stratification for treatment. ${ }^{27}$

In recent years, new studies focused on the evaluation of VTE (including pulmonary embolism and deep venous thrombosis) have been published. Among Canadian patients with patients, the risk of VTE was 1.53 (95\% CI 1.16 to 2.01) in comparison with the general population, while among Swedish patients with AS, it was 1.4 (95\% CI 1.1 to 1.9 ), showing a $50 \%$ increased risk of this event compared with the general population. ${ }^{26} 2829$ This increased risk could be explained by the chronic production of inflammatory cytokines (such as TNF-alpha and IL-16) and CRP, which leads to a hypercoagulable state.

\section{Cardiovascular risk factors}

CV risk factors can be classified as 'modifiable' (smoking, obesity, hypertension, diabetes and dyslipidaemia) and 'non-modifiable' (age, gender and family history of CVD). The COMOSPA study showed hypertension as the most prevalent $\mathrm{CV}$ risk factor among patients with SpA $(33.5 \%, 95 \%$ CI 32 to 35$) .{ }^{3}$ However, this prevalence is even higher among patients with PsA, ranging from $20 \%$ to $47 \%$ depending on the cohort. ${ }^{30-32}$ The prevalence of dyslipidaemia is also higher among patients with SpA in comparison with the general population $(19.1 \%$ vs $12.7 \%$, respectively), rising to $50 \%$ in some PsA US cohorts. $^{32} 33$ This greater prevalence of CV risk factors among patients with PsA in comparison with other populations could be explained by the well-known association between cutaneous psoriasis and metabolic disorders. ${ }^{34}$ Smoking seems to be the second most prevalent risk factor for CV disease in patients with SpA $(29.3 \%$, CI 27.9 to 30.4). Moreover, a higher prevalence of smokers has been described among patients with SpA with an axial phenotype than among those with a peripheral phenotype, ${ }^{11}$ which is associated with structural progression and ankylosis. ${ }^{35}$

\section{$\mathrm{SpA}$ treatment and CVD}

SpA treatment can also play a role in the increase in CVD. NSAIDs are the cornerstone in the management of these patients. These drugs inhibit the cyclo-oxygenase (COX) enzyme, responsible for converting arachidonic acid into prostaglandins, which act as mediators of inflammation and pain. COX-1 is present under physiological conditions, while COX-2 is induced only under inflammatory and damaged tissue conditions. The inhibition of the COX-1 isoform leads to anti-aggregation and vasodilating status, while the inhibition of the COX-2 isoform leads to a pro-thrombotic and vasoconstricted status. ${ }^{36}$ In recent years, some studies have shown that the cardiovascular adverse effects of NSAIDs do not depend only on specific COX inhibition, demonstrating a similar vascular risk between cyclo-oxygenase-2 inhibitors and other nonselective NSAIDs. ${ }^{36}{ }^{37}$ Studies evaluating the effect of NSAIDs on CVD show contradictory results, depending on the study population and on the type of NSAIDs. In 2011, Bakland et alshowed an inverse association between 
mortality and NSAID use in patients with SpA. ${ }^{4}$ Similarly, another study showed a significant association between traditional NSAID use and CVD mortality reduction among patients with $\mathrm{SpA}$ aged 66 years or older. ${ }^{23} \mathrm{On}$ the other hand, Dubreuil et al demonstrated that current use of diclofenac was associated with MI in patients with SpA (OR 3.3, 95\% CI 1.57 to 7.0) regardless of age, while the association with the use of naproxen was not significant. ${ }^{33}$ These results suggest distinct effect of diverse NSAID regimens on CV disease, which could help clinical decision-making specially in patients at increased risk of cardiovascular disorders.

\section{Management of CVD}

The COMOSPA study provided knowledge not only for the prevalence of comorbidities but also for the evaluation of the gap between the available recommendations and their implementation in daily clinical practice. ${ }^{3}$ In fact, only $50.5 \%$ of patients were optimally monitored for CVD (all measurable risk factors for CV disease monitored at least once during the previous year), ${ }^{38}$ showing high variability among countries. Two risk prediction algorithms are usually used to calculate a 10-year risk of CVD events: the Framingham Risk Score (validated in the North American population) and the Systematic Coronary Risk Evaluation (SCORE) (validated in the European population). When the CVD risk evaluated with the Framingham Risk Score exceeds a 10 -year risk of $10 \%$ for fatal or non-fatal CVD events or when the SCORE exceeds a 10-year risk of $5 \%$ for fatal CVD events, lipid-lowering treatment and lifestyle changes are recommended. ${ }^{39}{ }^{40} \mathrm{In}$ 2017, the update of the 2015/2016 EULAR recommendations for cardiovascular disease management in patients with chronic inflammatory joint diseases (IJDs) was published. ${ }^{41}$ These recommendations emphasise the importance of optimally controlling disease activity to lower CVD risk in patients with IJD, since the number and duration of flares over time seems to contribute to the CVD risk. ${ }^{42}$ Moreover, according to these recommendations, CVD risk should be assessed at least once every 5 years, which is in line with the 2016 European Guidelines on cardiovascular disease prevention in clinical practice. ${ }^{43}$ Finally, CVD risk management should be conducted according to the national guidelines in patients with IJD, and NSAIDs in these patients should be prescribed with caution, especially in patients with previous CVD or in those with the presence of CVD risk factors.

\section{INFECTIONS}

Data on infections in SpA are scarce and come mainly from randomised controlled trials (RCTs). A few metaanalyses have evaluated the risk of serious infections in patients with SpA exposed and not exposed to biological treatment. In 2010, a meta-analysis of randomised placebo-controlled trials showed a risk of serious infection, between $0 \%$ and $0.9 \%$, in patients with SpA not exposed to anti-TNF, while the risk of serious infections in exposed patients ranged from $0 \%$ to $2.9 \%$, with the rate of serious infections being non-significant between both groups. ${ }^{44}$ Other recent meta-analysis showed similar results, confirming there is no increased risk of infections associated with anti-TNF therapy in these patients. ${ }^{45} 46$

\section{Management of infections}

The scarce serious infections reported in some studies were both viral and bacterial infections. Although these adverse events in patients with SpA are not very frequent, specific recommendations for vaccination for patients with rheumatic disease have been published. ${ }^{47}$ In these patients, vaccination should be preferably administered prior to planned immunosuppression, with seasonal influenza and pneumococcal vaccination strongly recommended. Despite these recommendations, the COMOSPA study demonstrated a non-optimal rate of vaccination in these patients, showing that only $17.3 \%$ received a pneumococcal vaccination within the past 5 years, and $30 \%$ received an influenza vaccination within the past 12 months. ${ }^{3}$ A recent clinical trial (COMEDSPA, NCT02374749) demonstrated that a nurse-led programme can improve the percentage of patients receiving vaccination; for patients who visited a nurse checking for comorbidities, $28.6 \%$ (influenza) and $40.0 \%$ (pneumococcal) received vaccinations, compared with $9.9 \%$ (influenza) and $21.1 \%$ (pneumococcal) of patients who did not have a comorbidity check by the nurse. $^{48}$

\section{MALIGNANT DISEASES}

In the COMOSPA study, the overall prevalence of any type of cancer was $3.0 \%$ (95\% CI 2.46 to 3.52 ), with cervical cancer being the most prevalent $(1.2 \%, 95 \%$ CI 0.3 to 1.7 ), which can be explained by the fact that specific cancer screening programmes in females are well established. ${ }^{3}$ However, some studies have reported that the risk for malignancy between patients with SpA and the general population is comparable. ${ }^{40}{ }^{50}$ The risk of colorectal cancer (CRC) is increased in patients with IBD, which can coexist with SpA; however, the increased risk of CRC in these patients has not been confirmed. ${ }^{51} \mathrm{An}$ increased risk of skin cancer has been reported in patients using p-UVA and UVB therapy, which is widely used in patients with psoriasis. ${ }^{52}$ However, studies concerning anti-TNF therapy have not reported an increased risk for malignant diseases in this population. A recent study including 8703 patients with SpA initiating a first anti-TNF therapy did not report an increased risk of cancer in exposed patients. ${ }^{49}$ These results were confirmed in a meta-analysis, which concluded the absence of an association between anti-TNF agents and cancer risk in patients with SpA. ${ }^{45}$

Patients with SpA with IBD may have a greater risk of gastrointestinal cancer. Crohn's colitis and ulcerative are associated with a high risk of CRC, while the risk of small bowel adenocarcinoma in patients with Crohn's enteritis 
is greatly elevated in comparison with the general population. ${ }^{53}$

\section{Management of malignant diseases}

Screening recommendations for patients with SpA are identical to the general population, except for IBDassociated CRC and skin cancer. Surveillance for colitisassociated dysplasia in patients with Crohn's disease should begin $8-10$ years after the diagnosis at intervals that are determined by risk factors (such as primary sclerosis cholangitis, pan-ulcerative colitis, active inflammation, pseudopolyps or a family history of colorectal carcinoma).${ }^{54}$ For skin cancer, a consensus based on systematic literature review and an expert consensus agreed that individuals ever exposed to DMARDs should visit a dermatologist one per year. ${ }^{55}$ Nevertheless, their implementation is not optimal. For example, the COMOSPA study showed that only $10.7 \%$ of patients exposed to bDMARDs were optimally screened for skin cancer. ${ }^{3}$ Moreover, only $32.7 \%$ and $44.0 \%$ of patients with SpA were in agreement with the recommendations for CRC and breast cancer prevention, respectively.

\section{FIBROMYALGIA}

Fibromyalgia (FM) is a chronic condition characterised by the presence of pain, as the dominant symptom, associated with fatigue, non-refreshed sleep, mood disturbance and cognitive impairment. ${ }^{56}$ The prevalence of FM in the general population ranges from $2 \%$ to $7 \%$, while in patients with $\mathrm{SpA}$, this percentage increases to $25 \% .{ }^{57} 58$ However, this prevalence varies depending on the application of the different FM criteria. Baraliakos et al demonstrated that the prevalence of coexistent FM using the 2010 criteria was significantly higher than that using the 1990 criteria (24\% vs $14 \%$, respectively) in the same cohort, ${ }^{59}$ while Molto et al demonstrated that the prevalence using the FiRST questionnaire ${ }^{60}$ was even higher $(37.8 \%)$, confirming that concomitant FM is more frequent in patients with SpA (especially in women with peripheral enthesitis) but not more than in other rheumatic diseases. ${ }^{61}$ The evaluation of $\mathrm{FM}$ in patients with $\mathrm{SpA}$ is of particular interest since the coexistence of this comorbidity leads to an impact on treatment and patient management. It has been demonstrated that concomitant FM has a significant impact on the antiTNF response after 12 weeks of follow-up, but only when this response is evaluated by patient-reported outcomes and not by objective biological parameters (ie, CRP). ${ }^{61}$ Moreover, patients with FM are more likely to switch to other anti-TNF treatments, and the retention rate of the first anti-TNF treatment is shorter in comparison with patients without FM. ${ }^{62}$

\section{Management of fibromyalgia}

The EULAR revised recommendations for the management of FM published in 2017 should be used in these patients. ${ }^{63}$ The overarching principles of these recommendations are, first, to comprehensively assess pain, function and psychosocial context; and, second, the objective of the management of FM is to improve healthrelated quality of life using a multidisciplinary approach that combines pharmacological and non-pharmacological treatment modalities. The first step is the use of exercise, given its effect on pain, physical function and well-being (without distinction between aerobic or anaerobic exercise). Meditative movement therapies or mindfulnessbased stress reduction are also strongly recommended, since they improve sleep and quality of life, as well as physical therapies (acupuncture or hydrotherapy). Hypnotherapy, massage and other alternative therapies are not recommended because of a lack of effectiveness. In the case of a lack of effect of the above non-pharmaceutical treatments, pharmacological therapies should be considered, especially for patients with severe pain (duloxetine, pregabalin, tramadol) or sleep disturbance (amitriptyline, cyclobenzaprine, pregabalin). NSAIDs, monoamine oxidase inhibitors, selective serotonin reuptake inhibitors and strong opioids are not recommended because of a lack of efficacy and the risk of side effects.

\section{Implementation of management of comorbidities in clinical} practice: the 'systematic review'

As we have previously exposed, recent studies have highlighted that a gap exists between recommendations for screening/management of comorbidities and their implementation in patients with $\mathrm{SpA}^{3}{ }^{64}$ Some have even suggested that their comorbidities management would be worse than the general population. One of the reasons might be the belief by general practitioners (GPs) (and even patients themselves) that rheumatologists are the leading physician in the management of these patients, including their comorbidities; furthermore, it is very unlikely for GPs to be familiar with all the specific recommendations for comorbidities management in patients with chronic rheumatic inflammatory diseases; finally, it is also possible that GP's are reluctant to prescribe some drugs or administer vaccines, particularly in patients treated with bDMARDs, for fear of drugreactions/interactions.

On the other hand, it is very unlikely that jobbing rheumatologists will find the time in their 10-15 min outpatient visit to also check for comorbidities with the currently available tools.

Nevertheless, recent studies have suggested that systematic screening of comorbidities improves its management. ${ }^{4865}$

In the light of these remarks emerged the idea of the 'systematic annual review' to be performed in all patients with chronic rheumatic inflammatory diseases, including SpA. This optimal management of patients with SpA includes a holistic approach which usually necessitates the collaboration of different health professionals (eg, rheumatologists, nurses, physiotherapists, ...). This approach has been recognised by international scientific societies ${ }^{66}$ and healthcare providers, ${ }^{67}$ by recommending 
a 'period systematic review': this review includes usually the clinical measurements needed to be repeated, educational programmes, evaluation of adherence to treatment and of course then systematic screening of comorbidities and their management.

Some data have suggested that a nurse intervention might be of great help in implementing this 'systematic review' and improve management of comorbidities. The COMEDSPA study demonstrated that the number of patients in agreement with recommendations for influenza and pneumococcal vaccination were significantly higher among patients who received a nurse intervention programme (28.6\% vs $9.9 \%$ and $40.0 \%$ vs $21.1 \%$ for influenza and pneumococcal vaccination, respectively). Similarly, skin cancer screening $(36.3 \%$ vs $17.2 \%$ for the active vs control group) and vitamin D supplementation initiation $(22.6 \%$ vs $8.7 \%)$ were more frequently performed in the active group. ${ }^{48}$

\section{CONCLUSIONS}

There is evidence of the high prevalence of comorbidities in patients with SpA, which leads to an important impact on the burden of disease and on healthcare resources. Osteoporosis is the most prevalent comorbidity in these patients and is mainly caused by systemic inflammation and a lack of mobility. CVD explains the increased mortality in patients with SpA in comparison with the general population, with atherosclerosis being one of the most important causes. Data on severe infections in daily clinical practice are scarce, but data from RCTs show a low incidence of infections both in patients with and without immunosuppressive treatment. There is no evidence of a high incidence of malignant diseases, except for skin cancer in patients with psoriasis who received p-UVA and UVB therapy. Concomitant FM deserves attention, since its coexistence with SpA leads to a poorer treatment response and more switches of anti-TNF treatments. Periodic holistic review for patients with $\mathrm{SpA}$, including systematic screening for comorbidities, might be the key to improve its management, and nurses might play an essential role in the implementation of such reviews.

\section{Contributors CL-M and AM contributed equally.}

Funding The authors have not declared a specific grant for this research from any funding agency in the public, commercial or not-for-profit sectors.

Competing interests None declared.

Patient consent for publication Not required.

Provenance and peer review Commissioned; externally peer reviewed.

Data availability statement This review was conducted using published data referenced in the main manuscript. No individual data from studies have been managed.

Open access This is an open access article distributed in accordance with the Creative Commons Attribution Non Commercial (CC BY-NC 4.0) license, which permits others to distribute, remix, adapt, build upon this work non-commercially, and license their derivative works on different terms, provided the original work is properly cited, appropriate credit is given, any changes made indicated, and the use is non-commercial. See: http://creativecommons.org/licenses/by-nc/4.0/.
ORCID iDs

Clementina López-Medina http://orcid.org/0000-0002-2309-5837

Anna Molto http://orcid.org/0000-0003-2246-1986

\section{REFERENCES}

1 Dougados M, Baeten D. Spondyloarthritis. Lancet 2011;377:2127-37.

2 López-Medina C, Moltó A. Update on the epidemiology, risk factors, and disease outcomes of axial spondyloarthritis. Best Pract Res Clin Rheumatol 2018;32:241-53.

3 Moltó A, Etcheto A, van der Heijde D, et al. Prevalence of comorbidities and evaluation of their screening in spondyloarthritis: Results of the international cross-sectional ASAS-COMOSPA study. Ann Rheum Dis 2016;75:1016-23.

4 Bakland G, Gran JT, Nossent JC. Increased mortality in ankylosing spondylitis is related to disease activity. Ann Rheum Dis 2011:70:1921-5.

5 Moltó A, Dougados M. Comorbidities in spondyloarthritis including psoriatic arthritis. Best Pract Res Clin Rheumatol 2018;32:390-400.

6 Baillet A, Gossec L, Carmona L, et al. Points to consider for reporting, screening for and preventing selected comorbidities in chronic inflammatory rheumatic diseases in daily practice: a EULAR initiative. Ann Rheum Dis 2016;75:965-73.

7 Briot K, Roux C. Inflammation, bone loss and fracture risk in spondyloarthritis. RMD Open 2015;1:e000052.

8 Briot K, Durnez A, Paternotte S, et al. Bone oedema on MRI is highly associated with low bone mineral density in patients with early inflammatory back pain: results from the DESIR cohort. Ann Rheum Dis 2013;72:1914-19.

9 Briot K, Etcheto A, Miceli-Richard C, et al. Bone loss in patients with early inflammatory back pain suggestive of spondyloarthritis: results from the prospective DESIR cohort. Rheumatology 2016;55:335-42.

10 Fechtenbaum M, Molto A, Roux C, et al. Baseline MRI inflammation is not a determinant of 5-year bone mineral density loss in patients with early spondyloarthritis. Joint Bone Spine 2020;87:63-8.

11 Glantsching H, Fisher JE, Wesolowski G, et al. M-CSF, TNF-alpha and RANK ligand promote osteoclast survival by signaling through mTOR/ S6 kinase. Cell Death Differ 2003;10:1165-77.

12 van der Weijden MA, van der Horst-bruinsma IE, van Denderen JC, et al. High frequency of vertebral fractures in early spondylarthropathies. Osteopor Int 2012;23:1683-90.

13 Maas F, Spoorenberg A, BPG VDS, et al. Clinical risk factors for the presence and development of vertebral fractures in patients with ankylosing spondylitis. Arthritis Care Res (Hoboken) 2017;69:694-702.

14 Ghozlani I, Ghazi M, Nouijai A, et al. Prevalence and risk factors of osteoporosis and vertebral fractures in patients with ankylosing spondylitis. Bone 2009;44:772-6.

15 Malochet-Guinamand S, Pereira B, Tatar Z, et al. Prevalence and risk factors of low bone mineral density in spondyloarthritis and prevalence of vertebral fractures. BMC Musculoskelet Disord 2017;18:357.

16 Sahuguet J, Fechtenbaum J, Molto A, et al. Low incidence of vertebral fractures in early spondyloarthritis: 5 -year prospective data of the DESIR cohort. Ann Rheum Dis 2019;78:60-5.

17 Campagna R, Pessis E, Feydy A, et al. Fractures of the ankylosed spine: MDCT and MRI with emphasis on individual anatomic spinal structures. AJR Am J Roentgenol 2009;192:987-95.

18 LES RHUMATISMES EN 100 questions. Available http://www.rhuma tismes.net/index.php? $p=1$ (accessed 20 Jan 2020)

19 Briot K, Geusens P, Em Bultink I, et al. Inflammatory diseases and bone fragility. Osteoporos Int 2017;28:3301-14.

20 Roubille C, Richer V, Starnino T, et al. Evidence-based recommendations for the management of comorbidities in rheumatoid arthritis, psoriasis, and psoriatic arthritis: expert opinion of the Canadian dermatology-rheumatology comorbidity initiative. $J$ Rheumatol 2015:42:1767-80.

21 Piepoli MF, Hoes AW, Agewal S, et al. 2016 European guidelines on cardiovascular disease prevention in clinical practice: the sixth joint task force of the European Society of Cardiology and other societies on cardiovascular disease prevention in clinical practice. Atherosclerosis 2016;252:207-74

22 Exarchou S, Lie E, Lindström U, et al. Mortality in ankylosing spondylitis: results form a nationwide population-based study. Ann Rheum Dis 2016;75:1466-72.

23 Haroon NN, Paterson JM, Li P, et al. Patients with ankylosing spondylitis have increased cardiovascular and cerebrovascular mortality: a population-based study. Ann Intern Med 2015;163:409-16.

24 González-Juanetey C, Vázquez-Rodríguez TR, Miranda-Filloy JA, et al. The high prevalence of subclinical atherosclerosis in patients with ankylosing spondylitis without clinically evident cardiovascular disease. Medicine (Baltimore) 2009;88:358-65. 
25 López-Medina C, Jiménez-Gómez Y, Moltó A, et al. Cardiovascular risk factors in patients with spondyloarthritis from Northern European and Mediterranean countries: an ancillary study of the ASAS-COMOSPA project. Joint Bone Spine 2018;85:447-53.

26 Eriksson JK, Jacobsson L, Bengtsson K, et al. Is ankylosing spondylitis a risk factor for cardiovascular disease, and how do these risks compare with those in rheumatoid arthritis? Ann Rheum Dis 2017;76:364-70.

27 Ogdie A, YiDing Y, Haynes K, et al. Risk of major cardiovascular events in patients with psoriatic arthritis, psoriasis and rheumatoid arthritis: a population-based cohort study. Ann Rheum Dis 2015;74:326-32.

28 Bengtsson K, Forsblad-d'Elia H, Lie E, et al. Are ankylosing spondylitis, psoriatic arthritis and undifferentiated spondyloarthritis associated with an increased risk of cardiovascular events? A prospective nationwide population-based cohort study. Arthritis Res Ther 2017;19:102

29 Aviña-Zubieta JA, Chan J, De Vera M, et al. Risk of venous thromboembolism in ankylosing spondylitis: a general population-based study. Ann Rheum Dis 2019;78:480-5.

30 Radner H, Lesperance T, Accortt NA, et al. Incidence and prevalence of cardiovascular risk factors among patients with rheumatoid arthritis, psoriasis or psoriatic arthritis. Arthritis Care Res (Hoboken) 2017;69:1510-18.

31 Barbarroja N, Arias-de la Rosa I, López-Medina C, et al. Cardiovascular risk factors in psoriatic disease: psoriasis versus psoriatic arthritis. Ther Adv Musculoskel Dis 2019;11:1-3.

32 Shah K, Paris M, Mellars L, et al. Real-world burden of comorbidities in US patients with psoriatic arthritis. RMD Open 2017;3:e000588.

33 Dubreuil M, Louie-Gao Q, Peloquin CE, et al. Risk of myocardial infarction with use of selected non-steroidal anti-inflammatory drugs in patients with spondyloarthritis and osteoarthritis. Ann Rheum Dis 2018;77:1137-42.

34 Gisondi P, Fostini AC, Fossà I, et al. Psoriasis and the metabolic syndrome. Clin Dermatol 2018;36:21-8.

35 Poddubnyy D, Haibel H, Listing J, et al. Cigarette smoking has a dose-dependent impact on progression of structural damage in the spine in patients with axial spondyloarthritis: results from the GErman SPondyloarthritis Inception Cohort (GESPIC). Ann Rheum Dis 2013;72:1430-2.

36 Braun J, Baraliakos X, Westhoff T. Nonsteroidal anti-inflammatory drugs and cardiovascular risk: a matter of indication. Semin Arthritis Rheum 2019 Aug 1.

37 Bhala N, Emberson J, Merhi A, et al. Coxib and traditional NSAID Trialists' (CNT) Collaboration Vascular and upper gastrointestinal effects of non-steroidal anti-inflammatory drugs: meta-analyses of individual participant data from randomised trials. Lancet 2013;382:769-79.

38 Peters MJL, Symmons DPM, McCarey D, et al. EULAR evidence-based recommendations for cardiovascular risk management in patients with rheumatoid arthritis and other forms of inflammatory arthritis. Ann Rheum Dis 2010;69:325-31.

39 D’Agostino RB, Vasan RS, Pencina MJ, et al. General cardiovascular risk profile for use in primary care: the Framingham Heart Study. Circulation 2008;117:743-53.

40 Conroy RM, Pyorala K, Fitzgerald AP, et al. Estimation of ten-year risk of fatal cardiovascular disease in Europe: the SCORE project. Eur Heart $J$ 2003;24:987-1003.

41 Agca R, Heslinga SC, Rollefstad S, et al. EULAR recommendations for cardiovascular disease risk management in patients with rheumatoid arthritis and other forms of inflammatory joint disorders: 2015/2016 update. Ann Rheum Dis 2017;76:17-28.

42 Arts EE, Fransen J, den Broeder AA, et al. The effect of disease duration and disease activity on the risk of cardiovascular disease in rheumatoid arthritis patients. Ann Rheum Dis 2015;74:998-1003.

43 Piepoli MF, Hoes AW, Agewall S, et al. 2016 European guidelines on cardiovascular disease prevention in clinical practice: the sixth joint task force of the European Society of Cardiology and other societies on cardiovascular disease prevention in clinical practice (constituted by representatives of 10 societies and by invited experts): Developed with the special contribution of the European Association for Cardiovascular Prevention \& Rehabilitation (EACPR). Eur Heart J 2016;37:2315-81.

44 Fouque-Aubert A, Jette-Paulin L, Combescure C, et al. Serious infections in patients with ankylosing spondylitis with and without TNF blockers: a systematic review and meta-analysis of randomised placebo-controlled trials. Ann Rheum Dis 2010;69:1756-61.
45 Hou LQ, Jiang GX, Chen YF, et al. The comparative safety of TNF inhibitors in ankylosing spondylitis: a meta-analysis update of 14 randomized controlled trials. Clin Rev Allergy Immunol 2018;54:234-43.

46 Wang S, He Q, Shuai Z. Risk of serious infections in biological treatment of patients with ankylosing spondylitis and non-radiographic axial spondyloarthritis: a meta-analysis. Clin Rheumatol 2018;37:439-50.

47 Furer V, Rondaan C, Heijstek MW, et al. 2019 update of EULAR recommendations for vaccination in adult patients with autoimmune inflammatory rheumatic diseases. Ann Rheum Dis 2020;79:39-52.

48 Moltó A, Gossec L, Poiraudeau S, et al. Evaluation of the impact of a nurse-led program of systematic screening of comorbidities in patients with axial spondyloarthritis: the results of the COMEDSPA prospective, controlled, one year randomized trial. Semin Arthritis Rheum 2020;50:701-8.

49 Hellgren K, Dreyer L, Arkema EV, et al. Cancer risk in patients with spondyloarthritis treated with TNF inhibitors: a collaborative study from the ARTIS and DANBIO registers. Ann Rheum Dis 2017;76:105-11.

50 Rohekar S, Tom BDM, Hassa A, et al. Prevalence of malignancy in psoriatic arthritis. Arthritis Rheum 2008;58:82-7.

51 Feltelius N, Ekbom A, Blomqvist P. Cancer incidence among patients with ankylosing spondylitis in Sweden 1965-95: a population based cohort study. Ann Rheum Dis 2003;62:1185-8.

52 Archier E, Devaux S, Castela E, et al. Carcinogenic risks of psoralen UV-A therapy and narrowband UV-B therapy in chronic plaque psoriasis: a systematic literature review. J Eur Acad Dermatol Venereol 2012;26:22-31.

53 Cannon J. Colorectal neoplasia and inflammatory bowel disease. Surg Clin North Am 2015;95:1261-9.

54 Roda G, Chien Ng S, Kotze PG, et al. Crohn's disease. Nat Rev Dis Primers 2020;6:22.

55 Gossec L, Baillet A, Dadoun S, et al. Collection and management of selected comorbidities and their risk factors in chronic inflammatory rheumatic diseases in daily practice in France. Joint Bone Spine 2016;83:501-9.

56 Häuser W, Ablin J, Fitzcharles MA, et al. Fibromyalgia. Nat Rev Dis Primer 2015;13:15022.

57 Wolfe F, Ross K, Anderson J, et al. The prevalence and characteristics of fibromyalgia in the general population. Arthritis Rheum 1995;38:19-28.

58 Zhao SS, Duffield SJ, Goodson NJ, et al. The prevalence and impact of comorbid fibromyalgia in inflammatory arthritis. Best Pract Res Clin Rheumatol 2019;33:101423.

59 Baraliakos X, Regel A, Kiltz U, et al. Patients with fibromyalgia rarely fulfil classification criteria for axial spondyloarthritis. Rheumatology 2018;57:1541-7.

60 Perrot S, Peixoto M, Dieudé P, et al. Patient phenotypes in fibromyalgia comorbid with systemic sclerosis or rheumatoid arthritis: Influence of diagnostic and screening tests. Screening with the FIRST questionnaire, diagnosis with the ACR 1990 and revised ACR 2010 criteria Clin Exp Rheumatol 2017;35:35-42.

61 Moltó A, Etcheto A, Gossec L, et al. Evaluation of the impact of concomitant fibromyalgia on TNF alpha blockers' effectiveness in axial spondyloarthritis: results of a prospective, multicentre study. Ann Rheum Dis 2018;77:533-40.

62 Bello N, Etcheto A, Béal C, et al. Evaluation of the impact of fibromyalgia in disease activity and treatment effect in spondyloarthritis. Arthritis Res Ther 2016;18:42.

63 Macfarlane GJ, Kronisch C, Dean LE, et al. EULAR revised recommendations for the management of fibromyalgia. Ann Rheum Dis 2017;76:318-28.

64 Dougados M, Soubrier M, Antunez A, et al. Prevalence of comorbidities in rheumatoid arthritis and evaluation of their monitoring: results of an international, cross-sectional study (COMORA). Ann Rheum Dis 2014;73:62-8.

65 Dougados M, Soubrier M, Perrodeau E, et al. Impact of a nurse-led programme on comorbidity management and impact of a patient self-assessment of disease activity on the management of rheumatoid arthritis: results of a prospective, multicenter, randomized, controlled trial (COMEDRA). Ann Rheum Dis 2015;74:1725-33.

66 Kiltz U, RBM L, van der Heijde D, et al. Development of ASAS quality standards to improve the quality of health and care services for patients with axial spondyloarthritis. Ann Rheum Dis 2020;79:193-201.

67 Quality statement 5: Annual review | rheumatoid arthritis in over 16s | quality standards | NICE. Available https://www.nice.org.uk/guidance/ qs33/chapter/Quality-statement-5-Annual-review (accessed 9 Mar 2020) 